\title{
The Einstein's Frog
}

\author{
Jauro COLLAÇO ${ }^{1}$
}

The $38^{\text {th }}$ Brazilian Congress of Cardiovascular Surgery, held in Porto Alegre, Brazil, from March 31 to April 2, 2011, and its Symposia, in particular Biotronik, held on April 1, 2011, reminded me the metaphor attributed to Einstein: "“If you put a frog in a pot with water and the temperature is increasing slowly, the frog does not notice, it will get used and will die. But if you put the frog into a pan of hot water, he immediately realizes the shock and bounce off'. I believe that we, cardiovascular surgeons, we were in the first pot, wake up and gradually started to jump out when we felt the heat of the second pot.

After moment of introspection, I came upon a latent need to write what follows.

All domestic and foreign speakers, without exception, made exciting presentations. I take the liberty to make mention of some of these notables, as well as to present my opinion on the matters contained in the official program.

Full Professor Domingo Marcolino Braile, from São José do Rio Preto, spoke on the surgeon's journey, since the days of the barber-surgeon to become a cardiovascular surgeon, returning to the writing of Arthur C. Clarke, adapted for the classic film by Stanley Kubrick's "2001: A Space Odyssey", what we were, who we are and what we think will be translated by one tibia thrown into space by a primitive primate, transformed into a space station, from where we went to a source of research represented by the enigmatic futuristic pyrolytic monument.

The foreign guests brought back, with minor changes, the lectures from European and American Congress of 2010 and the Royal Society in November 2010 in London.

I understand that although the media have evolved rapidly in recent years and the World Wide Web (Internet) has become indispensable as a channel of information, the physical presence of the speaker is still essential for the interaction between experts. Perhaps in the near future, tele or video conferencing will evolve and could replace physical meetings. This is extremely important because the fees, rates and materials paid for by SUS - Unified Health System - and the majority of agreements do not allow all members of a surgical team to participate in an international congress, whether it be here in Brazil or abroad.

The presentations in Porto Alegre showed the participants what the world thinks about our specialty and that takes time to make sure that the results are reliable.
The Heart Team has existed for many years, because in the 60's when I got involved with cardiac surgery and went to the Clinics Hospital in São Paulo, all patients with heart disease, one suspects that the surgery could help, underwent a daily medical-surgical discuss, under the aegis of Full Professor EJ Zerbini, and on Wednesdays, with the presence of Professor. Alípio Correa Neto. The English term is more recent, from the early 2000s, and gained momentum when the SYNTAX trial began to be formulated and was summarized to the clinical and interventional cardiologistsurgeon.

In the wake of these events, cardiovascular surgery teams started to participate in the Heart Teams for electrical treatment of heart disease, heart failure, valvular and vascular stents. The frog felt the warmth of the water.

The meeting of medical and surgical specialists to decide the best treatment in the light of current knowledge, is mandatory. However, each must have the detachment to accept the national and international guidelines and more recent, like many of medical, clinical, surgical and interventional cardiologists, are physician-dependent, not everyone has the ability to perform a particular procedure, as well as follow the progress of their patients. The cardiologists persists in its mission to respond, diagnose, indicate the best treatment in conjunction with the Heart Team and also keep the patient under their observation to feel that it is progressing satisfactorily as decided.

Therefore, the application of trials, as COURAGE, MASS II, BARI 2D and SYNTAX, among others, should really be considered with extreme caution by the experts responsible for the area, checking those who have more competence.

Full Professor Pieter Kappetein, Rotterdam, Holland, presented again its statement of November 2010 at the Royal Society in London in Valve Technology Symposium, or that is, the idea of SYNTAX- LIKE for TAVI (Transcatheter Aortic Valve Implantation), a fact which I considered splendid because he is a surgeon who represents the future of our specialty, because he is aware of what is happening in the East and West European countries with smaller or larger economic conditions similar to those of South America. The idea is valid for any percutaneous or similar procedures in the heart, great vessels, supra-aortic or peripheral arteries.

Full Professor David Taggart, Oxford, UK, has warned 
that the analyses of trials on the treatment of obstructive coronary disease are not always correct. The medical and surgical treatments have evolved in recent years and interventional cardiology, although it has undergone several changes, need more observation time to be indicated as an initial procedure in chronic patients.

The interventions on the coronary arteries decreased, according to the Centers for Medicare \& Medicaid Services of the United States, demonstrating a national trend between 2001 and 2009.

Full Professor Enio Buffolo, São Paulo, SP, insisted on the doctor-patient relationship, especially when we live in, with so many treatment options. He believes that the patient should be given all the information about the advantages and disadvantages of each method to be used, to the classics that are still in clinical trials.

The development of stem cells and equivalent deserves more careful. Besides high cost, the ethical and technical vibrant discussions between adult and embryonic cells refer, according to the centers of our knowledge and literature, results for long-term outcomes. Therefore, it must be the persistence on the study without showing off early miracles.

Treatment of CHF by electrical treatment, is more complex. I still follow the postulates of $\mathrm{PhD}$ Patricia F. Bakker, University of Utrecht, Holland, a pioneer of resynchronization with the left ventricle via the electrode under the control of epicardial echocardiography. It deserves to point out that my first patient to receive a resynchronization was a 29 -year-old woman, who had refused her transplant in Brazil and the United States of America. She returned to my care, and then, armed with all the exams, I went to Utrecht to discuss the case with Dr. Bakker. She advised me to deploy a resynchronization epicardial lead and, since the surgery, the patient is today (10 years) out of the transplant list in functional class II. The discussion on which electrode or endocardial or epicardial electrodes should be implemented remains. The research on the apical endocardial electrode introduced in the left ventricle should continue.

The 2D video-assisted surgery, with wide application in the abdomen, thorax, orthopedics and urology, was introduced clinically for the heart by Full Professor Alain Carpentier, France, on February 26, 1996. One should not to forget to mention the experience of Full Professor Hugo Vanermen, Belgium, among others, that did not evolve the expected in these 15 years, utilizing CPB, cannulation of peripheral vessels, transthoracic aortic clamping or endoclamps, cardioplegia, prolonged and costly surgical time. The syndrome of acute inflammatory response was not controlled, despite the efforts of various groups, in particular the Hammersmith Hospital in London, led by pioneer Full Professor Kenneth Taylor.

Full Professor Cornelius Borst, Utrecht, Holland, was a tireless researcher on the proposals for off-pump cardiac surgery and the insistence that the coronary arteries should be handled as little as possible, but also promoted the first demonstrations of video-assisted cardiac surgery and 2D and $3 \mathrm{D}$ robotic, for 12 years. It is likely that the surgical repair of atrial fibrillation, pericardial disease, implantation of epicardial leads or dissection of the internal thoracic arteries, 2D video-assisted surgery has a more comprehensive space in the future. If performed in $3 \mathrm{D}$, it may be not less than the traditional surgery in some situations, since the instrumental technology is advancing rapidly. In this respect, one must consider cost/benefit.

The new valve endoprostheses that can also be deployed under direct vision with smaller incisions may become more interesting for so-called minimally invasive surgery, as has been researched in Poland, Germany and Switzerland, among others.

Mechatronics, which has invaded all areas of medicine, did not show in the last 20 years, especially in cardiovascular surgery, initially with Zeus and currently with the Da Vinci advantage over existing techniques. Both the surgical limitations, similar to laparoscopic surgery (heart as a body mass, three-dimensional, requiring several views, and therefore multiple entries in the left hemithorax or right or both), and the cost. It is a line of research that should not be abandoned, after all, Full Professor Volkmar Falk, currently in Switzerland, has the experience needful to Medicine.

The so-called hybrid operating rooms are in fact normal cardiovascular operating rooms to the present day. Those demonstrated in Congress are not the most modern. Those equipped with the latest generation of surgical tables and C-three-dimensional arcs, with the possibility of coronary angiography and hemodynamic studies of the heart or systemic vessels, will allow performing virtual procedures, demonstrated by the Japanese for years, as do the pilots of modern aircraft, as the Airbus A380 for takeoff and landing.

Returning to the surgical teams, one must understand that if cardiovascular surgeons to survive have to specialize in echocardiography, electrophysiology, basic science and molecular medicine, among other areas, one should not forget that the cardiovascular surgeon has to be complete, that is, he is primarily a surgeon, who devoted himself to a complex specialty.

Patients nowadays referred for surgery, are more severe, progressively elderly (beyond 80 years), valve reoperations or CABG (1st, 2nd, 3rd, 4th), revascularizations after intrastents obstructions, renal, pulmonary, obstructions of the supra-aortic and aortic disease, making it a challenge for surgeons. Therefore, surgical teams need to understand that there is need for the surgeon who spends all day working to have time to drink water or juice and eat a snack, while a colleague and the anesthesiologist monitor the 
patient, precisely because the surgeries have become more extensive and elaborate than those of the 70s of last century.

There is a conference earlier this week, I was in the operating room after surgery, when a general surgery's resident called from emergency unit at the Hospital and asked if there was a cardiac surgeon to attend him. As I had already left the field, I decided to answer him and he told me he was patient with stab wound in the precordium, the left hemithorax was drained ( $300 \mathrm{ml}$ of blood), but CT of the chest had shown a slide fluid, probably blood in the pericardial sac. I went to the emergency room to see the wounded and observed an individual torpor, albeit with normal vital signs and saturating above $90 \%$. Physical examination revealed two wounds faster than $3 \mathrm{~cm}$ in length, penetrating the square of Ziegler and paradoxical pulse. I sent immediately to the operating room and called the general surgery residents to observe. To summarize, the knife had entered the 4th left intercostal space, passed through the chest wall, pericardium, transfixed the right ventricle, the anterior wall to the bottom and through the injury through the pericardium, justadiafragma, I introduced the finger and felt that the abdomen had been penetrated. I opened the abdominal wall, the cavity of which had large amounts of blood, and found that the left lobe of the liver was transfixed, also the omentum and part of the stomach wall. It was corrected the need in the abdomen, diaphragm and heart, and there was no lung injury. The patient, a garbage collector, was discharged from hospital 3 days after surgery.
Undoubtedly, there are few hospitals in Brazil that have emergency room capable of servicing a patient under the conditions described, as well as a cardiovascular surgical team ready and available. Currently, there are few surgeons who are dedicated to the surgical center full time, requiring surgical teams to define which of its components must be eligible for a subspecialty.

However, not all surgeons have or may have all the technological wonders that exist or arise. The surgeons on active duty, even young ones, can not only think they are late because they can not use certain techniques such those described at Symposia in the Congress and programming in general. One should understand that traditional cardiovascular surgeon is a surgeon complete, a team which includes the subspecialties of Cardiovascular Surgery Team.

If this thought is followed, the two goals of surgical treatment will be achieved, without doubt: relieve symptoms and improve patients' survival .

I am absolutely sure that the barber-surgeon was looking for the same ant the Einstein's frog is awakening some time ago.

1 - IV Adjunct Professor of the Thoracic and Cardiovascular Surgery Discipline at Federal University of Santa Catarina (UFSC). Professor of Cardiac Surgery of the University of South Santa Catarina (UNISUL). Head of the Cardiovascular Surgery Service of the Cardiology Institute of Santa Catarina State.

E-mail: jaurocollaco@yahoo.com.br 